\title{
RESUMEN GRÁFICO DE LA HETEROGENEIDAD DE LAS ACTITUDES EN UN DESTINO TURÍSTICO
}

Ramón Cardona, José ${ }^{1}$

Serra Cantallops, Antoni ${ }^{2}$

Universitat de les Illes Balears

jose.ramon@uib.es

antoni.serra@uib.es

Material original autorizado para su primera publicación en la revista académica REDMARKA. Revista Digital de Marketing Aplicado.

http://doi.org/10.17979/redma.2012.02.09.4754

Recibido: 3 Noviembre 2012

Aceptado 15 Diciembre 2012

\section{RESUMEN}

En las últimas décadas, diversos estudios empíricos han segmentado las actitudes de los residentes hacia el turismo. Pueden observarse similitudes en estos estudios, llevando a especular que podrían existir tipologías predefinidas cuyo peso dependería de la fase del ciclo de vida en que se encuentre el destino turístico. De la revisión y análisis de las segmentaciones existentes en la literatura se puede proponer una Segmentación Teórica, aparentemente válida para diferentes destinos turísticos. La Segmentación Teórica sugiere cinco grupos, define sus perfiles, propone su peso relativo, y su evolución prevista a lo largo del ciclo de vida. La Segmentación Teórica resume los estudios empíricos existente y proporciona indicaciones generales para los gestores, en ausencia de estudios empíricos específicos.

\footnotetext{
${ }^{1}$ Doctorando del programa de economía de empresa de la Universidad de les Illes Balears. Profesor asociado del área de Comercialización e Investigación de Mercados en la Universidad de les Illes Balears.

2 Profesor Titular del área de Comercialización e Investigación de Mercados en la Universidad de les Illes Balears. Investigador principal del grupo Dirección y Gestión de Empresas y Destinos Turísticos.
} 
A continuación, se plantea la interacción de la Segmentación Teórica propuesta con dos variables demográficas: el tiempo de residencia en la región y si el individuo es nativo o no. Estas dos variables permiten nuevos desgloses con mayor detalle y claridad en la descripción de la población. Este nuevo desglose genera una elevada cantidad de información que puede ser resumida gráficamente.

En la segunda parte del trabajo, se realiza un estudio empírico en la isla de Ibiza que busca poner a prueba la viabilidad de la Segmentación Teórica propuesta y la representación gráfica de la información. El análisis clúster genera una segmentación que concuerda en gran medida con la Segmentación Teórica. Las únicas diferencias observadas parecen debidas a la fuerte crisis que padecía la isla en el momento del trabajo de campo. Las diferentes variantes de representación gráfica realizadas resumen de forma visual los resultados principales del análisis, aunque necesitan mejoras metodológicas.

Palabras Clave: actitudes de los residentes, segmentación, gráfico.

\section{ABSTRACT}

GRAPHICAL OVERVIEW OF ATTITUDES HETEROGENEITY IN A TOURIST DESTINATION

In recent decades, several empirical studies have segmented residents' attitudes towards tourism. Similarities can be observed in these studies, leading to speculate that there might be weighing predefined types depending on the phase of the life cycle of the destination. From a review and analysis of the existing literature on segmentations a Theoretical Segmentation can be proposed being apparently valid for different destinations. This Theoretical Segmentation suggests five groups, define their profiles, relative weight and anticipated changes throughout the life cycle. Theoretical Segmentation summarizes existing empirical studies and provides general guidelines for managers in the absence of specific empirical studies.

As a second step, we analyze the interaction of the proposed Theoretical Segmentation with two demographic variables: time of residence in the region and if the individual is native or not. These two variables allow further 
breakdowns in greater detail and clarity in the description of the population. This new breakdown generates a large amount of information that can be summarized graphically.

In the second part of the paper, we carry out an empirical study on the island of Ibiza that seeks to test the feasibility of the proposed Theoretical Segmentation and graphic representation of information. The cluster analysis generates a segmentation that corresponds closely with the Theoretical Segmentation. The only differences observed seem to be due to the severe crisis that afflicted the island at the time of the fieldwork. The different graphics outlined visually summarize the main results of the analysis, although there is still a need for methodological improvements.

Keywords: residents' attitudes, segmentation, graphical. 


\section{INTRODUCCION}

El objetivo de la segmentación es describir una población mediante su división en grupos homogéneos y el análisis de sus respuestas promedio (Williams y Lawson, 2001). La segmentación es una aproximación a la situación ideal, que es el análisis de de cada individuo. Habitualmente los estudios sobre actitudes de los residentes consideran a la población local homogénea y analizan las relaciones causa-efecto que generan la respuesta media (Allen, Hafer, Long, y Perdue, 1993; Allen, Long, Perdue, y Kieselbach, 1988; Besculides, Lee, y McCormick, 2002; Gursoy, Chen, y Yoon, 2000; Gursoy, Jurowski, y Uysal, 2002; Mason y Cheyne, 2000; Perdue, Long, y Allen, 1990; Teye, Sirakaya, y Sönmez, 2002). Pero es razonable pensar que existe una heterogeneidad en las actitudes de los residentes. Hay modelos que proponen una evolución de las actitudes de los residentes, como el de Doxey (1975) y el de Butler (1980), pero consideran que las actitudes son homogeneas en un momento dado y variables en el tiempo, y también existe heterogeneidad en un momento dado (Belisle y Hoy, 1980). En las últimas décadas se han realizado estudios empíricos que buscan segmentar los residentes, con la finalidad de detectar grupos distintos según su actitud hacia el turístico:

- Una de las primeras segmentaciones la encontramos en un de la peninsula de Sleat (Isla de Skye), en Escocia (Brougham, 1978; Brougham y Butler, 1981).

- En el trabajo de Davis, Allen y Cosenza (1988) en Florida se identificaron cinco grupos: los Lovers (20\%), los Haters (16\%), los Cautious Romantics (20\%), los In-Betweeners (18\%) y los Love 'em for a Reason (26\%).

- Canan y Hennessy (1989) encontraron importantes diferencias en las actitudes de los residentes de Moloka'i (Hawaii), y Schroeder (1992) detecto tres grupos en Flagstaff (Arizona).

- En el estudio de Evans (1993) realizado en Nueva Zelanda se determinaron cuatro grupos: los Lovers (20\%) tienen un elevado nivel de renta y pertenecen a los Pakeha (descendientes de europeos); los Haters (11\%) tienen una amplia proporción de personas viudas, divorciadas o separadas, 
personas sin empleo o retirados y no-Pakeha (Maorís o grupos étnicos del Pacífico); los Controlled (32\%) son mayoritariamente retirados o con empleo y Pakeha, y los Selfish (37\%) son en su mayoría hombres, jóvenes, solteros, estudiantes y no-Pakeha. Se encontraron diferencias estadísticamente significativas en relación a las variables demográficas.

- El trabajo de Ryan y Montgomery (1994) en Bakewell (Inglaterra) se basa en el de Davis, Allen y Cosenza (1988) y usa una muestra muy reducida. Tres grupos fueron detectados: los Enthusiast (22\%), los Somewhat Irritated (24\%) y los Middle-of-the-Roaders (54\%). Las variables demográficas no eran estadísticamente significativas a excepción de la presencia de niños en la familia y el tiempo que se lleva residiendo en la población.

- En el estudio de Madrigal (1995) se comparaban dos destinos (Sedona y York). Fueron encontrados tres grupos: los Haters (31\%), los Lovers (13\%) y los Realists (56\%). Los Realists no muestran interés en participar en discusiones sobre planificación turística. Detecta mayores diferencias entre grupos que entre ciudades.

- Ryan, Scotland, y Montgomery (1998) detectaron en Rangitikei (Nueva Zelanda) tres grupos de residentes: los Moderate Enthusiasts (42,5\%), los Extreme Enthusiast (17,5\%) y los Cautious Supporters (40\%).

- El estudio de Fredline y Faulkner (2000) determina cinco grupos de residentes en relación a su actitud hacia el evento: los Ambivalent Supporter (Cautious Romantics) (29\%) viven fuera de la zona afectada por el evento; los Haters (15\%) son residentes que se oponen a la continuidad del evento y viven en la región desde hace más de 20 años; los Realists (24\%) trabajan en turismo o en sectores dependientes del turismo; los Lovers (23\%) son grandes defensores del evento, y los Concerned for a Reason (9\%) muestran preocupación por asuntos puntuales.

- En el estudio de Weaver y Lawton (2001) se detectaron tres grupos: los Supporters (27\%), los Opponents (22\%) y los Neutrals (51\%). Los Opponents son de edad más avanzada, residen en la región desde hace 
más tiempo que el resto y el motivo de su llegada a la región es la búsqueda de un lugar tranquilo.

- El trabajo de Williams y Lawson (2001) analiza las actitudes en diez ciudades de Nueva Zelanda. En su estudio detectaron cuatro grupos: los Lovers (45\%) son personas casadas, con casa propia y con mayor renta; los Cynics (10\%) son más viejos, tienen menos renta, menos personas casadas y menos propietarios de viviendas; los Taxpayers (25\%) residen en la ciudad desde hace menos de dos años, y los Innocents (20\%) son personas que viven en zonas con muy poca presencia turística.

- Thyne y Lawson (2001) realizaron un estudio de Southern Lakes Region, en Nueva Zelanda, y obtuvieron cuatro grupos: Lovers (14\%), We Miss Out (40\%), Self-Interest Supporters (30\%) y Critics (16\%).

- En un estudio realizado en Creta, Andriotis y Vaughan (2003) detectaron tres grupos: los Advocates (42,5\%), los Socially and Environmentally Concerned $(39,5 \%)$ y los Economic skeptics (18\%).

- En el estudio de Aguiló y Rosselló (2005) definen cinco grupos: los Development Supporters (11\%) creen que hay más oportunidades económicas; los Prudent Developers (26\%) creen que existen más oportunidades de trabajo, aunque se ha producido un cambio cultural importante; los Ambivalent and Cautious (24\%) presentan una opinión bastante ambigua; los Protectionists (20\%) son los más preocupados por los aspectos negativos y creen que el balance global es negativo, y los Alternative Developers (18\%) son partidarios de nuevos proyectos de desarrollo turístico.

- Brida, Osti y Barquet (2010) detectaron cuatro grupos: los Environmental Supporters (40\%) están de acuerdo con los impactos positivos económicos y socioculturales, y con los impactos negativos medioambientales; los Development Supporters (27\%) están de acuerdo con los impactos positivos del turismo y en desacuerdo con los impactos negativos; los Protectionists (14\%) están de acuerdo con los impactos negativos medioambientales y en 
desacuerdo con los impactos positivos, y los Ambivalents (18\%) tienen una postura ambigua.

En estos trabajos se detecta siempre la presencia de dos grupos con proporciones variables: los defensores y los detractores acérrimos. Además, los diversos estudios detectan entre uno y tres grupos adicionales. Los paralelismos son difíciles de realizar ya que suelen mostrar posturas menos claras que los otros dos grupos aunque tienden hacia posturas generales positivas, en su mayoría. Todo ello lleva a plantear una Segmentación Teórica que puede servir de referencia a los gestores de los destinos turísticos.

En este trabajo se plantea una Segmentación Teórica y su interacción con dos variables demográficas (el tiempo de residencia en la región y si el individuo es nativo o no). Estas dos variables permitirían nuevos desgloses que buscan un mayor detalle en la descripción de los residentes. Tras este planteamiento se procede a la realización de un estudio empírico que busca mostrar la viabilidad de la propuesta teórica realizada y plantear una forma de representación gráfica de la información obtenida: el Mapa de Actitudes.

\section{PROPUESTA TEÓRICA}

Al revisar los estudios empíricos publicados se observan similitudes, siendo posible establecerse una Segmentación Teórica que resume la heterogeneidad de la población residente en cinco grupos:

- Partidarios Entusiastas (del 10\% al 25\%). Está formado por las personas que reciben, o esperan recibir, de una forma directa los impactos positivos del turismo, por ejemplo trabajadores y empresarios del sector con elevadas rentas.

- Partidarios con Opinión Matizada (del 20\% al 60\%). Aumenta su presencia con el desarrollo turístico y está relacionado con la constatación de la dependencia turística regional. Sus opiniones son positivas pero matizadas por el reconocimiento de los impactos negativos. 
- Partidarios Interesados (del $10 \%$ al $35 \%$ ). Es inicialmente insignificante pero crece al aparecer inmigración atraida por la demanda de mano de obra. En este caso apoyan el turismo por intereses personales, concretamente por los ingresos económicos que aporta.

- Críticos (del $10 \%$ al $25 \%$ ). Suelen rondar el $10 \%$, pero en destinos muy masificados o mal gestionados sobrepasará el $20 \%$. Estos individuos no perciben beneficios directos del turismo pero si los costes.

- Sin Opinión Formada (del 0\% al 60\%). En los destinos maduros este grupo es muy pequeño. Sus opiniones son ambiguas y tienen un bajo contacto con el sector turístico.

La Segmentación Teórica plantea la posibilidad de que se den hasta cinco grupos claramente diferenciados por sus actitudes. No tienen porque aparecer en todas las regiones los cinco grupos. Si estarán los Partidarios Entusiastas y lo Críticos. Pero los otros tres grupos oscilarán enormemente o incluso desaparecerán en según qué casos. Los individuos Sin Opinión Formada serán la inmensa mayoría en los inicios del turismo, junto con un grupo de Partidarios Entusiastas que actuaran de motor de desarrollo. En las primeras fases de desarrollo turístico los individuos Sin Opinión Formada irán integrándose en los Partidarios Entusiastas o los Partidarios con Opinión Matizada. Las personas que resulten marginadas de los beneficios y sufran los costes pasarán a formar parte de los Críticos. En la fase de madurez los Partidarios Entusiastas representan una parte pequeña de la población. Si el nivel de dependencia de la región es muy elevado serán mayoría los Partidarios con Opinión Matizada. Dependiendo de la planificación y grado de masificación que sufra la región en su evolución, será mayor o menor el grupo de los Críticos.

Esta Segmentación Teórica aporta un primer desglose pero es posible analizar la interacción de esta clasificación con dos parámetros que suelen resultar significativos: el tiempo de residencia en la región (Aguiló y Rosselló, 2005; Brida, Osti y Barquet, 2010; Ryan y Montgomery, 1994; Weaver y Lawton, 2001; Williams y Lawson, 2001) y si el individuo es nativo o no (Aguiló y Rosselló, 2005; Canan y Hennessy, 1989; Davis, Allen y Cosenza, 1988; Evans, 1993; Madrigal, 1995). Parece ser que estas variables actúan como 
aproximación de los elementos personales que afectan de forma directa a las actitudes. Estas dos variables permitirían pasar de las segmentaciones actuales a nuevas dimensiones de desglose con mayor descripción de los residentes. Tomando como base estas variables demográficas se puede proponer una clasificación de los residentes en los siguientes grupos:

- El primer grupo estaría formado por nativos de la región. Este grupo lleva toda la vida residiendo en la región y desciende de personas nativas. Poseen un conocimiento muy amplio de la cultura local y la sienten como propia. Suelen tener actitudes favorables hacia el turismo en el inicio de la actividad turística. El grado de euforia depende del nivel económico local y la forma de implantar la actividad turística. Con el desarrollo del turismo la actitud de los nativos del lugar sufre cambios. Los que han alcanzado prosperidad económica tienen una opinión ampliamente favorable hacia el sector turístico, pero los habitantes que casi no han obtenido mejoras individuales y sufren los problemas tienen una opinión poco favorable o desfavorable.

- El siguiente grupo está formado por los residentes que no nacieron en la región pero que se trasladaron a vivir a la región en sus inicios. Este grupo no tiene como suya la cultura de la región pero la admira y conoce. Son personas que conocían la cultura y el modo de vida local, y decidieron abandonar su anterior residencia para vivir en un ambiente distinto. Se trata de personas más satisfechas con el modo de vida tradicional que los nativos. Se trata de un grupo más conservacionista que el anterior y que ofrece resistencia a las innovaciones que conlleva el desarrollo turístico y que llevaría hasta su casa los elementos de los que había escapado en su lugar de origen.

- Otro grupo serían los recién llegados. Residen desde hace poco tiempo en la región y han llegado impulsadas por las perspectivas económicas. Suelen ser personas jóvenes que viven solas o familias jóvenes. Su prioridad es el beneficio económico, siendo los costes sociales poco relevantes. Los costes más importantes para ellos son los costes económicos (nivel de precios, impuestos) y la inseguridad ciudadana. Este grupo de personas son las 
causantes de que haya una gran rotación de población en los destinos turísticos.

- Existe un grupo de residentes que llevan mucho tiempo en la región. En este caso se trata de personas que llegaron buscando una mejora económica personal y que han permanecido en ella de forma indefinida. Con el paso del tiempo su comportamiento va asemejándose al de los nativos y forman el núcleo no nativo de la población. Al adoptar las pautas de actitudes de los nativos, los residentes antiguos se dividen en dos grupos: los que han conseguido una próspera situación económica gracias al sector turístico poseen actitudes muy entusiastas, y los que han conseguido trabajo pero de nivel retributivo modesto y no han dispuesto de mejores oportunidades ni dentro ni fuera de la región tienen una actitud indiferente o negativa del turismo.

Tabla 1: Modelo de Mapa de Actitudes.

\begin{tabular}{|c|c|c|c|c|c|c|c|}
\hline \multirow{2}{*}{\multicolumn{2}{|c|}{$\begin{array}{l}\text { Destino Turístico } \\
(100 \%)\end{array}$}} & \multicolumn{4}{|c|}{ Con Opinión Formada y Clara (\%) } & \multicolumn{2}{|c|}{ Sin Opinión Clara (\%) } \\
\hline & & $\begin{array}{c}\text { Partidarios } \\
\text { Entusiastas } \\
(\%)\end{array}$ & $\begin{array}{c}\text { Partidarios } \\
\text { con Matices } \\
(\%)\end{array}$ & $\begin{array}{c}\text { Partidarios } \\
\text { Interesados } \\
(\%)\end{array}$ & Críticos (\%) & $\begin{array}{l}\text { Sin Opinión } \\
\text { Formada (\%) }\end{array}$ & $\begin{array}{c}\text { Neutros o } \\
\text { Moderados } \\
\text { (\%) }\end{array}$ \\
\hline \multirow{7}{*}{ 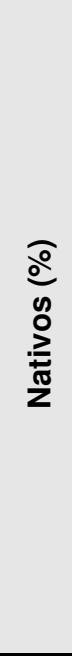 } & $\begin{array}{l}\text { Menos de a } \\
\text { años (\%) }\end{array}$ & $(\%)$ & $(\%)$ & $(\%)$ & $(\%)$ & $(\%)$ & $(\%)$ \\
\hline & $\begin{array}{l}\text { Entre a y b } \\
\text { años (\%) }\end{array}$ & $(\%)$ & $(\%)$ & $(\%)$ & $(\%)$ & $(\%)$ & $(\%)$ \\
\hline & $\begin{array}{c}\text { Entre b y c } \\
\text { años (\%) }\end{array}$ & $(\%)$ & $(\%)$ & $(\%)$ & $(\%)$ & $(\%)$ & (\%) \\
\hline & $\begin{array}{c}\text { Entre c y d } \\
\text { años (\%) }\end{array}$ & $(\%)$ & $(\%)$ & $(\%)$ & $(\%)$ & (\%) & (\%) \\
\hline & $\begin{array}{c}\text { Entre d y e } \\
\text { años (\%) }\end{array}$ & $(\%)$ & $(\%)$ & $(\%)$ & $(\%)$ & $(\%)$ & $(\%)$ \\
\hline & $\begin{array}{l}\text { Entre e y } f \\
\text { años (\%) }\end{array}$ & $(\%)$ & $(\%)$ & $(\%)$ & $(\%)$ & $(\%)$ & $(\%)$ \\
\hline & $\begin{array}{l}\text { Más de f } \\
\text { años (\%) }\end{array}$ & $(\%)$ & $(\%)$ & $(\%)$ & $(\%)$ & $(\%)$ & $(\%)$ \\
\hline \multirow{4}{*}{ 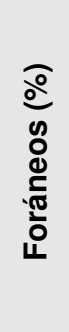 } & $\begin{array}{c}\text { Menos de g } \\
\text { años (\%) }\end{array}$ & $(\%)$ & $(\%)$ & $(\%)$ & $(\%)$ & $(\%)$ & $(\%)$ \\
\hline & $\begin{array}{l}\text { Entre g y } \mathrm{h} \\
\text { años (\%) }\end{array}$ & $(\%)$ & $(\%)$ & $(\%)$ & $(\%)$ & $(\%)$ & $(\%)$ \\
\hline & $\begin{array}{l}\text { Entre h y i } \\
\text { años (\%) }\end{array}$ & $(\%)$ & $(\%)$ & $(\%)$ & $(\%)$ & (\%) & $(\%)$ \\
\hline & Entre i y j & $(\%)$ & (\%) & $(\%)$ & $(\%)$ & $(\%)$ & (\%) \\
\hline
\end{tabular}




\begin{tabular}{|c|c|c|c|c|c|}
\hline años (\%) & & & $(\%)$ & $(\%)$ \\
\hline $\begin{array}{c}\text { Entre jy k } \\
\text { años (\%) }\end{array}$ & $(\%)$ & $(\%)$ & $(\%)$ & $(\%)$ \\
\hline $\begin{array}{c}\text { Más de k } \\
\text { años (\%) }\end{array}$ & $(\%)$ & $(\%)$ & $(\%)$ & $(\%)$ & $(\%)$ \\
\hline
\end{tabular}

Fuente: elaboración propia.

- Existe un grupo formado básicamente por gente joven. Forman este grupo jóvenes nacidos después del desarrollo turístico. Se trata de personas que han vivido siempre en un entorno muy turístico y han asimilado el estereotipo de la región. Estas personas enfocan su existencia al ocio y a las actividades típicamente turísticas. Su actitud hacia el turismo es muy ambigua.

La población residente puede dividirse de dos formas, según la opinión expresada o según las características demográficas que posean sus miembros, tal y como se ha comentado. Pero se puede ir un paso más allá de esta división teórica mediante la intersección de ambas segmentaciones. El resultado es una tabla o Mapa de Actitudes con frecuencias relativas y resume las actitudes de los residentes en una región y momento concretos en relación a una variable demográfica, permitiendo realizar comparaciones entre regiones y momentos distintos (Tabla 1). El dato contenido en cada una de las casillas de la tabla es el porcentaje de la muestra que posee una determinada actitud y una determinada categoría de la variable demográfica. De los segmentos de actitudes, uno de los dos de la parte de Sin Opinión Clara desaparecerá y en la parte Con Opinión Formada y Clara puede desaparecer uno de los grupos de partidarios moderados o fusionarse ambos. En todo caso, es muy recomendable mantener siempre las mismas divisiones en las aplicaciones empíricas para facilitar las comparaciones en el tiempo (estudios realizados en un destino pero en momentos del tiempo distintos) y en el espacio (comparar diversos destinos en un mismo momento). También es posible crear otros mapas de actitudes cruzando los grupos de actitudes con otras variables demográficas que resulten significativas y compliquen la representación gráfica tradicional. 
A esta tabla y a sus representaciones gráficas le llamamos Mapa de Actitudes, por el aspecto del gráfico usado. Puede representarse como una tabla, reforzada con colores, o como una imagen bidimensional o tridimensional, similar a un mapa topográfico, sintetizando en una imagen la composición de la sociedad según sus actitudes y características demográficas. Esta técnica de representación es una aplicación a pequeña escala de las técnicas usadas en áreas biomédicas para representar un gran volumen de información de forma intuitiva. En el siguiente apartado se realiza un estudio empírico que pone a prueba la Segmentación Teórica y el uso del Mapa de Actitudes como resumen de la información generada.

\section{ESTUDIO EMPÍRICO}

Ibiza es una isla del Mediterráneo con una fuerte dependencia económica del turismo y que recibe cerca de 2,000.000 de turistas al año. En la actualidad se encuentra en la fase de Estancamiento del Ciclo de Vida del Destino Turístico. El primer paso para conocer la estructura de la comunidad local es la realización de una segmentación de los residentes según sus actitudes hacia el turismo. De los diversos métodos de segmentación existentes, el más usado es el análisis clúster (Brida, Osti, y Barquet, 2010; Davis, Allen, y Cosenza, 1988; Fredline y Faulkner, 2000; Madrigal, 1995; Ryan y Montgomery, 1994; Schroeder, 1992; Weaver y Lawton, 2001; Williams y Lawson, 2001). En este caso se ha optado por la realización de una segmentación de los residentes mediante un análisis clúster jerárquico descendente (Algoritmo de HowardHarris) mediante el programa DYANE 4 (Santesmases, 2009), conservando la segmentación mayor que cumpla dos criterios: en todos los grupos hay al menos un $10 \%$ de la muestra contenido, y una nueva segmentación explicaría menos del $2 \%$ de la varianza.

Para la realización de esta segmentación se ha tomado como punto de partida un conjunto de 60 ítems que miden diversos aspectos de las actitudes de los residentes hacia el turismo. Estos ítems utilizaban una escala Likert de 5 puntos (Maddox, 1985), siendo 1 "Muy en Desacuerdo", 3 "Indiferente" y 5 "Muy de Acuerdo", pero con el añadido de una sexta opción de "No sabe / No 
contesta" (Weaver y Lawton, 2001). El trabajo de campo se realizó entre febrero y marzo de 2011 y como resultado se obtuvieron 418 encuestas válidas, obtenidas mediante un procedimiento aleatorio de selección. Tras la segmentación, se realizó el cálculo de la tabla ANOVA correspondiente y la tabulación cruzada de los grupos con las variables demográficas. El resultado es la generación de cinco segmentos, y la definición de sus características.

El grupo de los Partidarios Entusiastas (19,6\%). Este grupo muestra las opiniones más positivas hacia el turismo. Respecto al total de la muestra, tiene más hombres, personas más viejas y menos individuos que lleven toda la vida en la isla. En este grupo hay muchos empresarios y profesionales y pocos empleados y estudiantes. Es el grupo con la mayor proporción de personas que trabajan en contacto con turistas y son los que consideran en mayor grado que sus ingresos bajarían mucho en caso de crisis turística.

Son las personas con la imagen más positiva de los impactos económicos. Aunque consideran el turismo de la isla de baja calidad, su postura es la más benévola de la muestra. Son los más partidarios de que, gracias al turismo, han mejorado los servicios públicos y se ha generado oferta lúdica de interés para los residentes. Consideran la cultura local un elemento dinámico y cambiante. Poseen la percepción más positiva de los impactos del turismo sobre la sociedad y la cultura local. Consideran que el turismo no ha incrementado la delincuencia e inseguridad y no hay conflicto cultural. Además, el turismo favorece la conservación y no degrada medioambientalmente la isla.

En cuanto a la evolución futura, este grupo es partidario de un aumento en el volumen de turistas. Son el único grupo que se opone a las tasas turísticas. Creen que tienen capacidad para influir en el futuro de la isla. Consideran que el turismo genera beneficios para todos y se reparten de forma muy igualitaria. Son partidarios de que el turismo lo regule la administración pero también deben participar en la toma de decisiones los empresarios. Consideran que se vive mejor ahora que hace 20 años y están muy orgullosos de que tanta gente quiera visitar la isla. Globalmente, el turismo ha tenido impactos positivos y negativos, pero los positivos superan con mucho a los negativos, y la calidad de vida se ha elevado mucho. Finalmente hay que decir que los miembros de 
este grupo están muy convencidos de que el turismo tiene que seguir siendo la principal actividad económica.

El grupo de los Críticos (23,5\%). Este grupo está formado por las personas con peor valoración del turismo. Hay menos empresarios que en el resto de grupos y más empleados y estudiantes. Es el grupo con menos personas trabajando en contacto con turistas y, consecuentemente, el grupo que se considera menos afectado en sus ingresos familiares por una posible crisis turística. Este grupo usa el ibicenco más que la media $(73,5 \%)$.

Poseen la postura más crítica con la generación de inflación y el pago de impuestos y tasas, sólo superada ligeramente por el grupo de los Partidarios Interesados. Es el grupo que considera con mayor intensidad que el turismo es de baja calidad. Tienen la opinión mayoritaria de que no han mejorado los servicios públicos, ni cuantitativamente ni cualitativamente. Es el grupo con peor opinión de los impactos del turismo sobre la sociedad y la cultural local. Ven un conflicto cultural con inmigrantes y turistas. Son los más críticos con los impactos medioambientales del turismo y consideran que el turismo causa degradación y saturación del medioambiente.

En cuanto a la evolución futura, se oponen a seguir aumentando el número de turistas, ven bien que se mantenga el nivel actual y son los únicos que estarían de acuerdo con una reducción progresiva. Son los más partidarios de las tasas turísticas. Se oponen a la abertura de nuevas áreas de servicios turísticos, pero sobretodo se oponen a la creación de nuevas plazas de alojamiento. Son los que valoran más que se tenga en cuenta la opinión de la población, pero creen que no tienen capacidad para influir en el futuro de la isla. Consideran que el turismo genera beneficios pero el reparto es muy desigual. Son partidarios de que el turismo lo regule la administración y sin la participación de los empresarios en la toma de decisiones, ya que los consideran con excesiva influencia política. Consideran que no se vive mejor ahora que hace 20 años y son los que se sienten menos orgullosos de que tanta gente quiera visitar la isla. Globalmente, el turismo ha tenido impactos positivos y muchos impactos negativos, siendo los negativos superiores a los positivos, aunque reconocen que la calidad de vida ha mejorado algo gracias al turismo. Finalmente hay que 
decir que consideran que el turismo no debe de seguir siendo la principal actividad económica, aunque no es una postura rotunda.

El Grupo de los Partidarios Interesados (20,3\%). Perciben con fuerza los impactos negativos del turismo pero apoyan el desarrollo turístico por su elevado nivel de preocupación económica. Es el grupo con menor uso del ibicenco en su entorno familiar (55,3\%). Es el grupo más de acuerdo con la afirmación de que el turismo genera inflación y mayores tasas e impuestos. Son los más partidarios de la existencia de conflicto cultural, tanto con turistas como con inmigrantes.

Creen que tienen capacidad para influir en el futuro de la isla. Consideran que el turismo genera beneficios para todos pero se reparten de forma desigual. Son partidarios de que el turismo lo regule la administración pero también deben participar en la toma de decisiones los empresarios, a los que ven con mucha influencia política. Son el grupo más preocupado por la situación económica de la isla. Consideran que no se vive mejor ahora que hace 20 años pero se sienten muy orgullosos de que tantos turistas quieran visitar la isla. Globalmente, el turismo ha tenido impactos positivos y negativos, pero los positivos superan a los negativos y la calidad de vida ha aumentado bastante. Finalmente, opinan que el turismo tiene que seguir siendo la principal actividad económica.

El Grupo de los Partidarios con Opinión Matizada (23\%). Respecto a la media de la muestra, tiene más hombres, sus miembros son más viejos y hay más individuos que llevan toda la vida en la isla. Posee mayor proporción de funcionarios y empresarios y menor proporción de estudiantes, parados y empleados. En este grupo hay más personas trabajando en contacto con los turistas que la media y consideran que sus ingresos bajarían pero, en general, no de forma dramática. Este grupo tiene la mayor proporción de usuarios del ibicenco en su entorno familiar $(78,1 \%)$.

Su valoración de los impactos económicos del turismo es superior a la media y similar a la de los Partidarios Interesados. Consideran que hay una mejora cualitativa y cuantitativa de los servicios públicos. Poseen una valoración positiva de los impactos del turismo en la cultura y el patrimonio local, no 
considerando al turismo el causante de grandes cambios en la cultura local y mucho menos de la pérdida de las costumbres e identidad local. Consideran que no hay conflicto cultural con inmigrantes y turistas, pero esta opinión no es tan rotunda como la de los Partidarios Entusiastas.

De cara al futuro, consideran que se debe mantener el volumen actual de turistas y mejorar la calidad del turismo. Son moderadamente partidarios de las tasas turísticas. Creen que no tienen capacidad para influir en el futuro de la isla. Son los más partidarios de que la gestión se realice a nivel insular. Creen que el turismo genera beneficios para todos pero hay cierta desigualdad en el reparto. Son partidarios de que el turismo lo regule la administración y sin la participación de los empresarios en la toma de decisiones, ya que los consideran bastante influyentes políticamente. Les preocupa menos que a la media de la muestra la situación económica de la isla. Consideran que se vive mejor ahora que hace 20 años. Globalmente, el turismo ha tenido impactos positivos y negativos, pero los positivos superan a los negativos y la calidad de vida ha mejorado. Finalmente, opinan que el turismo tiene que seguir siendo la principal actividad económica.

El Grupo de los Neutros (13,6\%). Muestran opiniones ambiguas y confusas. Es el grupo con mayor presencia de mujeres, el más joven y el que tiene más individuos residiendo toda la vida en la isla. Tiene una gran proporción de empleados, estudiantes y parados y menor proporción de empresarios y profesionales liberales. En este grupo hay menos personas trabajando en contacto con los turistas que la media y consideran que una crisis turística no les afectaría o lo haría de forma moderada. Este grupo usa el ibicenco menos que la media $(57,9 \%)$.

Son el grupo con las valoraciones más bajas de los impactos económicos y su principal crítica es cualitativa no cuantitativa. Es el grupo con la postura más contraria a la idea de que el turismo satura los servicios e infraestructuras. Es el único grupo con una visión de la cultura local eminentemente estática.

Este grupo muestra una postura ambigua de cara al futuro, se oponen claramente a una disminución del volumen de turistas, pero son ambiguos al preguntarles por si sería adecuado aumentar o mantener el volumen de 
turistas. Son moderadamente partidarios de las tasas turísticas. Creen que no tienen capacidad para influir en el futuro de la isla. Consideran que se generan beneficios para todos pero se reparten de forma desigual. Son partidarios de que el turismo lo regule la administración y sin la participación de los empresarios en la toma de decisiones, ya que los consideran bastante influyentes políticamente. Junto al grupo de los Partidarios con Opinión Matizada son los menos preocupados por la situación económica de la isla. Consideran que no se vive mejor ahora que hace 20 años. Globalmente reconocen impactos positivos y negativos, y consideran que los positivos son algo superiores a los negativos, pero no tienen claro si la calidad de vida ha mejorado. Finalmente, opinan que el turismo tiene que seguir siendo la principal actividad económica, aunque es el grupo que muestra una postura afirmativa más moderada.

La segmentación empírica obtenida encaja en gran medida con la Segmentación Teórica expuesta y las pocas variaciones existentes son debidas a la crisis económica existente cuando se realizó el trabajo de campo. El siguiente paso es cruzar la segmentación obtenida con las variables demográficas y representarlo mediante el Mapa de Actitudes. A priori, y en base a la literatura existente, parece que las variables más interesantes son los años de residencia y si se ha nacido en la región. El objetivo pretendido con la idea del Mapa de Actitudes es conseguir resumir la información más relevante del análisis descriptivo en una imagen lo más intuitiva y sencilla posible.

\section{Tabla 2: Mapa de Actitudes de Ibiza (datos).}

\begin{tabular}{|c|c|c|c|c|c|c|}
\hline \multirow{2}{*}{\multicolumn{2}{|c|}{$\begin{array}{c}\text { ISLA DE IBIZA } \\
2011 \\
(100 \%)\end{array}$}} & \multicolumn{4}{|c|}{ Con Opinión Formada y Clara (87,3 \%) } & \multirow{2}{*}{$\begin{array}{c}\text { Sin Opinión } \\
\text { Clara (12,7\%) } \\
\text { Neutros } \\
(12,7 \%)\end{array}$} \\
\hline & & $\begin{array}{c}\text { Partidarios } \\
\text { Entusiastas } \\
(20,4 \%)\end{array}$ & $\begin{array}{c}\text { Partidarios } \\
\text { con Matices } \\
(22,1 \%)\end{array}$ & $\begin{array}{c}\text { Partidarios } \\
\text { Interesados } \\
(20,8 \%)\end{array}$ & $\begin{array}{l}\text { Críticos } \\
(24,1 \%)\end{array}$ & \\
\hline \multirow{2}{*}{ 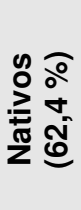 } & $\begin{array}{l}\text { Menos de } 25 \\
\text { años }(8,4 \%)\end{array}$ & $0,8 \%$ & $0,8 \%$ & $1,2 \%$ & $2,6 \%$ & $3,0 \%$ \\
\hline & $\begin{array}{c}\text { De } 25 \text { a } 34 \\
\text { años (13,7 } \\
\%)\end{array}$ & $2,2 \%$ & $1,8 \%$ & $3,2 \%$ & $3,0 \%$ & $3,6 \%$ \\
\hline
\end{tabular}




\begin{tabular}{|c|c|c|c|c|c|c|}
\hline & $\begin{array}{c}\text { De } 35 \text { a } 44 \\
\text { años }(11,6 \\
\%)\end{array}$ & $2,0 \%$ & $2,8 \%$ & $2,4 \%$ & $2,8 \%$ & $1,6 \%$ \\
\hline & $\begin{array}{c}\text { De } 45 \text { a } 54 \\
\text { años ( } 10,6 \\
\%)\end{array}$ & $1,6 \%$ & $5,0 \%$ & $2,2 \%$ & $1,6 \%$ & $0,2 \%$ \\
\hline & $\begin{array}{c}\text { Más de } 54 \\
\text { años }(5,8 \%)\end{array}$ & $2,2 \%$ & $1,8 \%$ & $0,6 \%$ & $1,2 \%$ & $0,0 \%$ \\
\hline \multirow{5}{*}{ 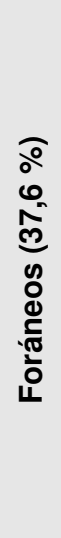 } & $\begin{array}{l}\text { Menos de } 5 \\
\text { años }(7,6 \%)\end{array}$ & $1,0 \%$ & $0,7 \%$ & $2,6 \%$ & $2,3 \%$ & $1,0 \%$ \\
\hline & $\begin{array}{c}\text { De } 5 \text { a } 14 \\
\text { años (15,6 } \\
\%)\end{array}$ & $4,0 \%$ & $2,6 \%$ & $3,6 \%$ & $4,6 \%$ & $0,7 \%$ \\
\hline & $\begin{array}{c}\text { De } 15 \text { a } 24 \\
\text { años }(6,6 \%)\end{array}$ & $1,3 \%$ & $1,7 \%$ & $1,0 \%$ & $2,0 \%$ & $0,7 \%$ \\
\hline & $\begin{array}{c}\text { De } 25 \text { a } 34 \\
\text { años (13,6 } \\
\%)\end{array}$ & $3,3 \%$ & $3,6 \%$ & $2,0 \%$ & $3,3 \%$ & $1,3 \%$ \\
\hline & $\begin{array}{c}\text { Más de } 34 \\
\text { años }(6,6 \%)\end{array}$ & $2,0 \%$ & $1,3 \%$ & $2,0 \%$ & $0,7 \%$ & $0,7 \%$ \\
\hline
\end{tabular}

Fuente: elaboración propia.

En la Tabla 2 se presentan los datos del Mapa de Actitudes de los residentes de Ibiza, tomando las dos variables demográficas mencionadas: años residiendo en la isla y si se ha nacido o no en ella ${ }^{3}$. La distribución por tramos de edad es muy parecida a los datos poblacionales. La proporción entre nacidos en la isla y nacidos fuera se ha corregido parcialmente con la finalidad de evitar un excesivo sesgo entre la muestra y la población, ya que los nacidos en lbiza han contestado en una proporción mayor que los nacidos fuera de la isla.

Figura 1: Mapa de Actitudes de Ibiza (imagen 2D).

${ }^{3}$ Existe una relación entre los años que llevan en la isla y la edad de los individuos significativa al 0,01 para la población foránea. Según los datos de la muestra, parece ser que la gran mayoría de las personas nacidas fuera de la isla se establecieron en ella a una edad comprendida entre los 20 y los 30 años. 


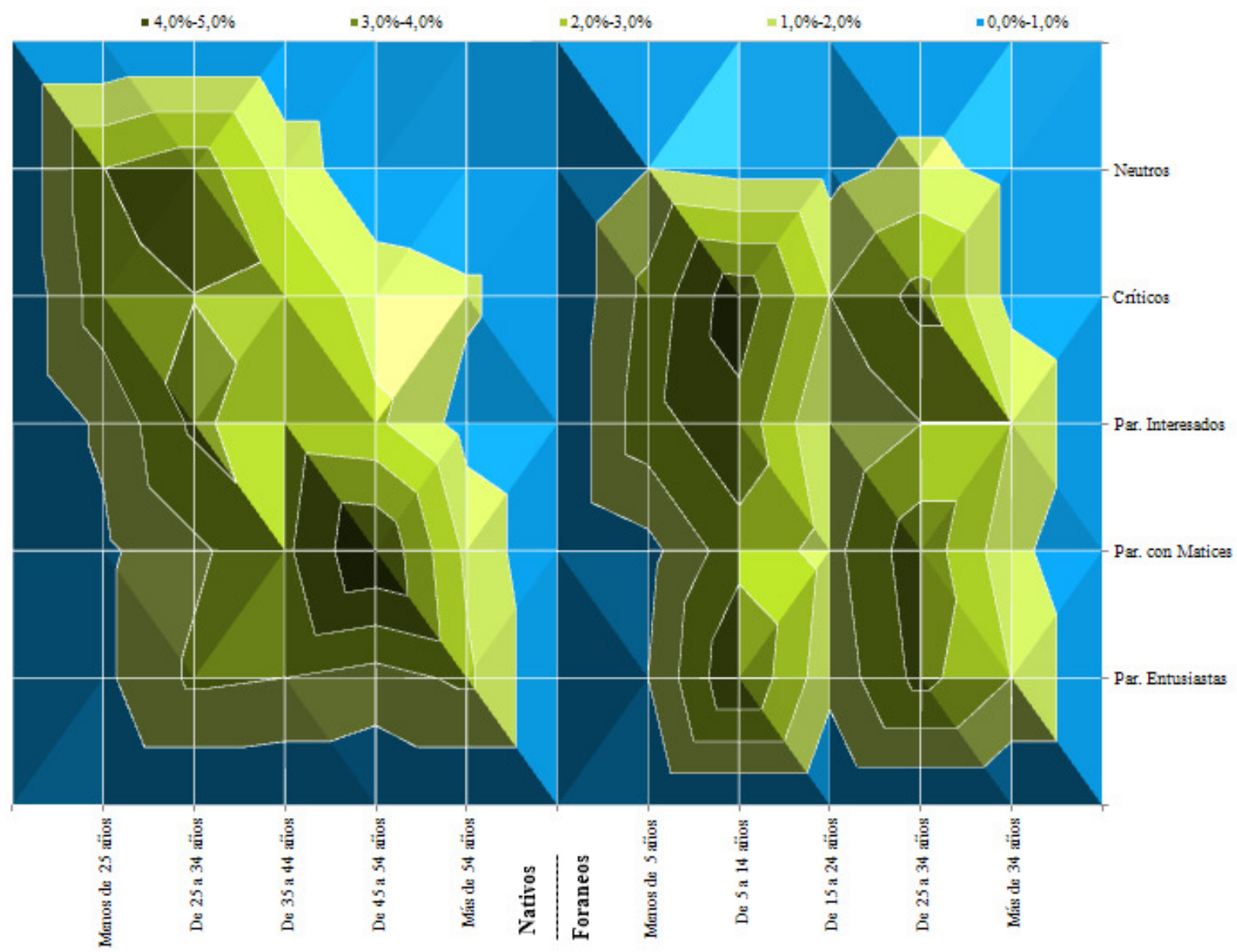

Fuente: elaboración propia.

En las Figuras 1 y 2 vemos la tabla del Mapa de Actitudes (Tabla 2) convertida una imagen semejante a un mapa topográfico. Las montañas muestran grupos de población que destacan por una combinación de variables demográficas y actitudes hacia el turismo. Los valles muestran grupos poco significativos, cuantitativamente hablando. Al realizar las tabulaciones cruzadas vemos que las personas nativas muestran actitudes significativamente distintas según el grupo de edad (parte izquierda de las figuras), pero las personas nacidas fuera no muestran diferencias significativas en sus actitudes en relación a los años residiendo en la isla (parte derecha de las figuras). Este hecho se puede observar en la existencia de una "formación montañosa" situada en diagonal en la parte izquierda y dos "cordilleras" paralelas en la parte derecha. 
Figura 2: Mapa de Actitudes de lbiza (imagen 3D).

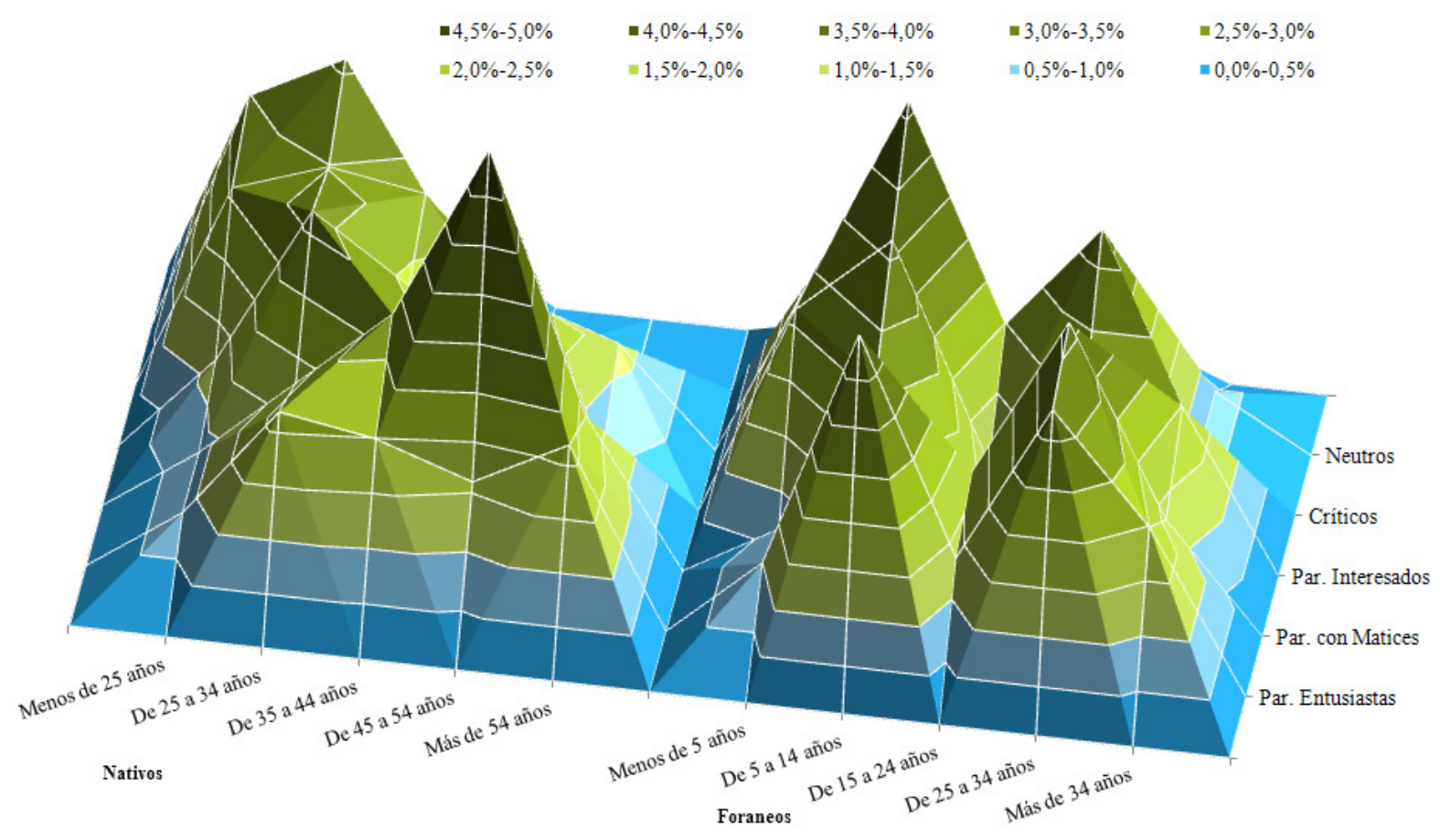

Fuente: elaboración propia.

La "formación montañosa" de la izquierda muestra que con la edad varía la actitud de los nacidos en la isla, siendo más positivas las personas mayores y más negativas o neutras los jóvenes. Las dos "cordilleras" de la derecha corresponden a personas nacidas fuera de la isla e indican que no son significativos los años que se lleve viviendo en la isla en la actitud hacia el turismo. El valle entre las dos "cordilleras" se debe a la crisis de finales de los ochenta y principios de los noventa. El resultado de esta forma de representación gráfica es que rápidamente se observa que:

- Los dos grupos más voluminosos son los nativos que rondan los 50 años y son Partidarios con Matices, y las personas que llegaron entre finales de los noventa y principios del siglo XXI y son Críticos con el sector.

- Los Neutros destacan por ser mayoritariamente personas de menos de 35 años nacidos en la isla. Posiblemente exista en la población de la isla un importante grupo de Neutros entre los inmigrantes llegados en los últimos años, pero este grupo es excepcionalmente reacio a participar en encuestas. 
- Los Partidarios Entusiastas son inmigrantes en su mayoría. Parece que entre los grandes beneficiados del sector turístico hay más personas foráneas que nativas.

- La crisis económica de finales de los ochenta y principios de los noventa se notó en la población, en especial entre las personas no nacidas en la isla. Este hecho lo vemos en el valle central de la formación de la derecha.

- Los Partidarios con Matices son mayoritariamente personas mayores y nacidas en la isla. La edad y, sobretodo, conocer la evolución de la isla a lo largo de muchos años facilita que se conozcan los pros y contras del turismo con gran profundidad.

- Las personas con pocos años en la isla son los más abundantes dentro de los Críticos con el turismo. Los recién llegados notan con fuerza los contrastes y peculiaridades de la isla en comparación con otras regiones.

De los grupos propuestos en el apartado teórico, en relación a las variables demográficas, vemos que: los nativos de mayor edad poseen una valoración positiva del turismo pero también son conscientes de costes; no se detecta la presencia de un grupo crítico de foráneos llegados en los inicios del turismo al tratarse de un destino maduro; entre los recién llegados hay muchos críticos y personas preocupadas por la situación económica; las personas llegadas hace más años parece que han evolucionado en sus opiniones hacia posturas parecidas a las de los nativos de similar edad pero no es un dato suficientemente significativo, y los jóvenes nacidos en la isla forman el grueso del grupo de los Neutros. Posiblemente la gestión de las actitudes de los residentes se pueda resumir en intentar conciliar la visión de los recién llegados críticos, con la visión de los nativos de más edad y partidarios con matices.

El Mapa de Actitudes podría crearse usando otras variables demográficas, pero en este estudio ninguna otra variable muestra las cualidades necesarias para que resulte interesante su uso: Ji cuadrado de la tabulación cruzada significativa y variable demográfica con suficientes categorías como para generar una imagen más visual con el Mapa que con otro tipo de gráfico más convencional. La mayoría de variables demográficas no muestran diferencias 
significativas en la tabulación cruzada con los grupos de actitudes. Las que muestran diferencias significativas son en su mayoría de dos o tres categorías. La única excepción es la variable edad, la cual cumple ambas condiciones.

\section{CONCLUSIONES}

La segmentación realizada concuerda en gran medida con la Segmentación Teórica propuesta a partir de la revisión de diversos estudios empíricos. Los Partidarios Entusiastas no son muy numerosos, situación esperable en destinos maduros. Las personas con pocos años en la isla son el grupo más abundante dentro de los Críticos. El porcentaje de población englobado en este grupo es muy superior al de destinos con un nivel de desarrollo menor, resultado esperable dada la literatura teórica existente (Doxey, 1975), pero podría ser superior a tenor de los resultados obtenidos en estudios realizados en otros destinos maduros y masificados. Los Partidarios con Opinión Matizada y los Partidarios Interesados poseen una actitud general positiva pero no tanto como los Partidarios Entusiastas. Los Partidarios con Opiniones Matizada son personas mayores y nacidas en la isla, en su mayoría. Los Partidarios Interesados son los menos integrados y con la peor situación económica, lo cual les genera preocupación y desencanto, sobre todo con la crisis económica. Finalmente, existe un grupo formado por persones sin una postura general clara, los Neutros, son mayoritariamente jóvenes de menos de 35 años nacidos en la isla. Los dos grupos más abundantes son los Críticos y los Partidarios con Opinión Matizada, lo cual es lógico si tenemos en un destino maduro. Por contra, el grupo minoritario son los Neutros.

Si avanzamos un paso más y cruzamos esta segmentación con las variables que habitualmente muestran una relación significativa con las actitudes (lugar de nacimiento y tiempo de residencia en el destino) podemos representar la información obtenida en un gráfico que hemos denominado Mapa de Actitudes. El Mapa de Actitudes es una evolución de la Segmentación Teórica planteada que busca aprovechar técnicas de representación de grandes volúmenes de datos, usadas en investigaciones biomédicas, para simplificar y clarificar los resultados principales de investigaciones descriptivas en ciencias sociales, en 
este caso actitudes de los residentes. En este trabajo se realiza un primer esbozo del Mapa de Actitudes y una aplicación práctica que permite mostrar en qué consiste el gráfico propuesto. La principal utilidad de esta herramienta es resumir la información de los estudios empíricos, de forma sencilla y clara, a los gestores de destinos turísticos.

Cabe indicar que sólo se ha planteado una primera idea y que necesita verificaciones y mejoras antes de convertirse en una herramienta que pueda resultar de aplicación rutinaria en los informes de análisis. Debe mejorarse la metodología para generar los datos del gráfico y el aspecto del gráfico. Además es necesario poner a prueba la técnica en diversos destinos turísticos antes de poder recomendar su uso rutinario.

\section{BIBLIOGRAFÍA}

Aguiló, E. \& Rosselló, J. (2005). Host Community Perceptions. A Cluster Análisis. Annals of Tourism Research, vol. 32, № 4, pp. 925-941.

Allen, L. R.; Hafer, H. R.; Long, P. T. \& Perdue, R. R. (1993). Rural residents' attitudes toward recreation and tourism development. Journal of Travel Research, vol. 32, no 1, pp. 27-33.

Allen, L. R.; Long, P. T.; Perdue, R. R. \& Kieselbach, S. (1988). The impact of tourism development on residents' perceptions of community life. Journal of Travel Research, vol. 27, no 1, pp. 16-21.

Andriotis, K. \& Vaughan, R. D. (2003). Urban residents' attitudes toward tourism development: The case of Creta. Journal of Travel Research, vol. 42, oㅡ 2, pp. 172-185.

Belisle, F. J. \& Hoy, D. R. (1980). The perceived impact of tourism by residents: A case study in Santa María, Colombia. Annals of Tourism Research, vol. 7, oㅜ 1, pp. 83-101.

Besculides, A.; Lee, M. \& McCormick, P. (2002). Resident's perceptions of the cultural benefits of tourism. Annals of Tourism Research, vol. 29, 끄 2, pp. 303-319.

Brida, G. G.; Osti, L. \& Barquet, A. (2010). Segmenting Resident Perceptions towards Tourism - a Cluster Analysis with a Multinomial Logit Model of a 
Mountain Community. International Journal of Tourism Research, vol. 12, ํㅜ 5, pp. 591-602.

Brougham, J. E. \& Butler, R. W. (1981). A segmentation analysis of resident attitudes to the social impact of tourism. Annals of Tourism Research, vol. 8, no 4, pp. 569-590.

Brougham, J. E. (1978). Resident attitudes towards the impact of tourism in Sleat. Disertación doctoral no publicada, University of Western Ontario (Canada).

Butler, R. W. (1980). The concept of a tourist area cycle of evolution: Implications for the management of resources. The Canadian Geographer, vol. 24, № 1, pp. 5-12.

Canan, P. \& Hennessy, M (1989). The growth machine, tourism and the selling of culture. Sociological Perspectives, vol. 32, pp. 227-243.

Davis, D.; Allen, J. \& Cosenza, R. M. (1988). Segmenting local residents by their attitudes, interests and opinions toward tourism. Journal of Travel Research, vol. 27, no 2, pp. 2-8.

Doxey, G. V. (1975). A causation theory of visitor-resident irritants: methodology and research inferences. En Conference Proceedings: Sixth Annual Conference of Travel and Tourism Research Association (San Diego), pp. 195-198.

Evans, T. R. (1993). Resident's perceptions of tourism in selected New Zealand communities: a segmentation study. Disertación de master, Dept. of Commerce, University of Otago.

Fredline, E. \& Faulkner, B. (2000). Host community reactions: A cluster analysis. Annals of Tourism Research, vol. 27, ํㅜ 3, pp. 763-784.

Gursoy, D.; Chen, J. S. \& Yoon, Y. (2000). Using structural equation modeling to assess the affects of tourism impact factors and local residents support for tourism development. Annual conference proceedings of the Travel and Tourism Research Association (Boise), pp. 243-250.

Gursoy, D.; Jurowski, C. \& Uysal, M. (2002). Resident attitudes: a structural Modeling Approach. Annals of Tourism Research, vol. 29, n 1, pp. 79105. 
Maddox, R. N. (1985). Measuring satisfaction with tourism. Journal of Travel Research, vol. 23, n 3, pp. 2-5.

Madrigal, R. (1995). Residents' perceptions and the role of government. Annals of Tourism Research, vol. 22, no 1, pp. 86-102.

Mason, P. \& Cheyne, J. (2000). Resident's attitudes to proposed tourism development. Annals of Tourism Research, vol. 27, no 2, pp. 391-411.

Perdue, R. R.; Long, P. T. \& Allen, L. (1990). Resident support for tourism development. Annals of Tourism Research, vol. 17, no 4, pp. 586-599.

Ryan, C. \& Montgomery, D. (1994). The attitudes of bakewell residents to tourism and numbers in comunity responsive tourism. Tourism Management, vol. 15, № 5, pp. 358-369.

Ryan, C.; Scotland, A. \& Montgomery, D. (1998). Resident attitudes to tourism development-A comparative study between the Rangitikei, New Zealand and Bakewell, United Kingdom. Progress in Tourism and Hospitality Research, vol. 4, oㅡ 2, pp. 115-30.

Santesmases, M. (2009). DYANE Versión 4. Diseño y Análisis de Encuestas en Investigación Social y de Mercados. Madrid: Ediciones Pirámide.

Schroeder, T. (1992). Host Community Perceptions of Tourism's impacts: A cluster analysis. Visions in Leisure and Business, vol. 10, no 4, pp. 43-48.

Teye, V.; Sirakaya, E. \& Sönmez, S. (2002). Resident's attitudes toward tourism development. Annals of Tourism Research, vol. 29, oㅜ 3, pp. 668-688.

Thyne, M. \& Lawson, R. (2001). Research Note: Addressing tourism public policy issues through attitude segmentation of host communities. Current Issues in Tourism, vol. 4, no 2-4, pp. 392-400.

Weaver, D. \& Lawton, L. (2001). Resident perceptions in the urban-rural fringe. Annals of Tourism Research, vol. 28, oㅡ 2, pp. 439-458.

Williams, J. \& Lawson, R. (2001). Community issues and resident opinions of tourism. Annals of Tourism Research, vol. 28, oㅡ 2, pp. 269-290. 